\title{
First Operational Experience from the LHCb Silicon Tracker
}

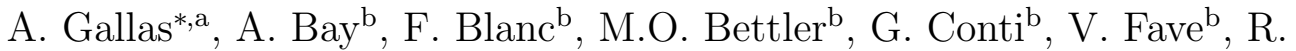

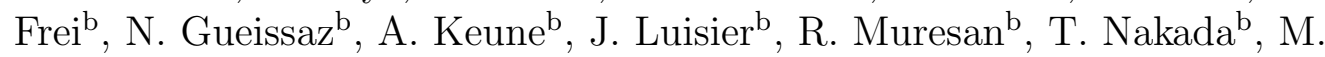 \\ Needham $^{\mathrm{b}}$, L. Nicolas ${ }^{\mathrm{b}}$, M. Knecht ${ }^{\mathrm{b}}$, A. Perrin ${ }^{\mathrm{b}}$, C. Potterat ${ }^{\mathrm{b}}$, O.

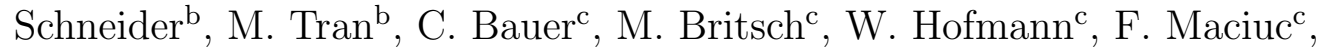 \\ M. Schmelling c, H. Voss ${ }^{\text {c }}$, J. Anderson ${ }^{\mathrm{d}}$, A. Buechler ${ }^{\mathrm{d}}$, N. Chiapolini ${ }^{\mathrm{d}}$, V. \\ Hangartner $^{d}$, C. Salzmann ${ }^{d}$, S. Steiner ${ }^{d}$, O. Steinkamp ${ }^{d}$, U. Straumann ${ }^{d}$, \\ M. Tobin ${ }^{\mathrm{d}}$, A. Vollhardt ${ }^{\mathrm{d}}$, B. Adeva ${ }^{\mathrm{a}}$, D. Esperante ${ }^{\mathrm{a}}$, A. Pazos-Alvarez ${ }^{\mathrm{a}}$, E. \\ Pérez-Trigo $^{a}$, P. Rodriguez Pérez ${ }^{\mathrm{a}}$, J. Saborido ${ }^{\mathrm{a}}$, V. Iakovenko ${ }^{\mathrm{e}}, \mathrm{O}$. \\ Okhrimenko $^{\mathrm{e}}$, V. Pugatch \\ ${ }^{a}$ University of Santiago de Compostela, Department of Particle Physics, Campus Sur, \\ Santiago de Compostela 15782, Spain \\ ${ }^{b}$ LPHE, EPFL Lausanne, 1015 Lausanne, Switzerland \\ ${ }^{c}$ Max Planck Institut für Kernphysik, 69117 Heidelberg, Germany \\ ${ }^{d}$ Physik-Institut der Universität Zürich, 8057 Zürich, Switzerland \\ ${ }^{e}$ National Academy of Sciences, Institute for Nuclear Research, 03680 Kiev, Ukraine
}

\begin{abstract}
The LHCb Silicon Tracker is a silicon micro-strip detector covering a sensitive area of $12 \mathrm{~m}^{2}$ with a total of $272 \mathrm{k}$ readout channels. The installation of the detector is complete and commissioning is making excellent progress. The detector has recorded first beam-induced events during LHC synchronization tests in August 2008 and in June 2009. These events have allowed the performance to be studied, and adjustments to the operational parameters to be made. In this contribution, we will draw first lessons from the in-situ commissioning of the Silicon Tracker, and present results from the reconstruction of data collected during the LHC synchronization tests.
\end{abstract}

Key words: LHC, LHCb, Silicon, Tracker, Semiconductor Detectors PACS: 07.77.Ka, 29.40.Wk

\footnotetext{
${ }^{*}$ Corresponding author. Tel.: +34-981-563100-14012; fax: +34-981-521091.

Email address: Abraham.Gallas@cern.ch (A. Gallas)
} 


\section{Introduction}

LHCb, a dedicated b-physics experiment at the LHC [1], will perform high-precision measurements of $\mathrm{CP}$ violation and rare decays of $\mathrm{B}$ hadrons. It is designed as a single-arm forward spectrometer as the production of $\mathrm{b}$ pairs is peaked a very low angle with respect to the beam axis.

The Silicon Tracker (ST) [2] consists of two detectors, both of which use long silicon micro-strips. The first, the Tracker Turicensis (TT), is located in the fringe field upstream of the LHCb $4 \mathrm{Tm}$ dipole magnet (Fig. 1). This $\sim 150 \mathrm{~cm}$ wide and $\sim 130 \mathrm{~cm}$ high planar tracking station covers the full detector acceptance. It has been constructed using p-on-n type sensors with a pitch of $183 \mu \mathrm{m}$ and a thickness of $500 \mu \mathrm{m}$ with the same design as in the CMS barrel tracker. It is composed of four layers arranged into two half stations separated $30 \mathrm{~cm}$ along the beam axis, and in orientation $0^{\circ}, 5^{\circ}$, $-5^{\circ}, 0^{\circ}$. Depending on the position in the layer up to four sensors are bonded together giving strip lengths up to $37 \mathrm{~cm}$.

The second of these detectors is the Inner Tracker (IT). It covers the region of highest particle density closest to the LHC beam pipe in the three $\mathrm{T}$ stations located downstream of the magnet. An IT station consists of four independent boxes arranged around the beam pipe in a $125 \mathrm{~cm}$ wide and $40 \mathrm{~cm}$ high cross shape (Fig. 1). Each box contains four layers of silicon micro-strips in orientation $0^{\circ}, 5^{\circ},-5^{\circ}, 0^{\circ}$. The ladders placed left and right of the beam pipe are $22 \mathrm{~cm}$ long with a thickness of $410 \mu \mathrm{m}$, the ladders above and below the beam pipe are $11 \mathrm{~cm}$ in length and with a thickness of 320 $\mu \mathrm{m}$, in both cases the read-out pitch is $198 \mu \mathrm{m}$.

The same front-end radiation hard read-out chip (the Beetle [3]) and read-out link [4] are used both in the IT and the TT. The TT (IT) has

been designed to withstand a radiation dose of 5 (8) x $10^{13} 1 \mathrm{MeV} \mathrm{n} / \mathrm{cm}^{-2}$ equivalent after 10 years of operation. Both detectors are operated at $5^{\circ} \mathrm{C}$.

\section{Installation and Commissioning}

The installation of the ST was completed by early summer 2008. At the time of the writing $99.7 \%$ of the channels in both IT and TT are fully operational. Commissioning with particles has started after the installation. 

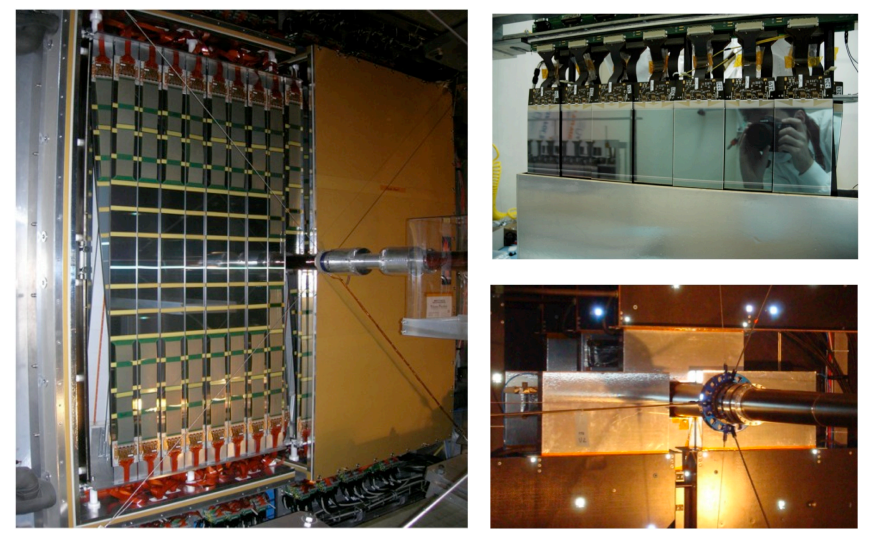

Figure 1: View of the TT installed in LHCb (left). View of the first IT station (bottom right), and detail of one of the detector boxes (top right).

The ST took part in the global LHCb cosmic running and the LHC synchronization described in the next section. This running allowed several faults, mainly related to lower power optical links, to be identified and repaired.

\section{Operation with particles: Performance studies}

Synchronization tests were performed during the start-up of the LHC machine at the end of August 2008 and the beginning of June 2009. A 450 $\mathrm{GeV}$ proton beam was extracted from the SPS and dumped onto a beam stopper (the 'TED') located $350 \mathrm{~m}$ downstream of LHCb. Monte Carlo simulations indicate the majority of particles reaching the Silicon Tracker are $10 \mathrm{GeV} / \mathrm{c}$ muons. With this data an initial time and spatial alignment for both the IT and TT detectors together with others has been made.

The first step during the TED runs was to perform an internal time alignment of the detector to take into account for the different lengths of the signal cables as well as the different time of flight of particles for the different stations. Runs were taken for both the IT and the TT where the delay between the sampling time and the trigger time was varied in 6.5 ns steps around the maximum of the signal, and the most probable value of the the signal determined for each set of ladders group in one front-end service box. The delay time for the subsequent data taking was then chosen as the one that maximized the most probable signal amplitude. After this procedure the detector is time aligned with a precision better than 1 ns. 
During the TED runs the particle density in the detectors was very high, more than 20 times the expected track density for a collision with a B meson. A robust stand-alone track reconstruction, capable of coping with this high track density, has been developed for the IT. This has been used together with the LHCb alignment software described in [5] to internally align the IT. These studies use a sample of 16000 isolated tracks selected from the TED events with the lowest occupancy. The detector boxes were aligned for translations in $\mathrm{X}$ (horizontal), $\mathrm{Y}$ (vertical) and rotations about the $\mathrm{Z}$ (beam) axis, the detector layers for $\mathrm{X}$ translations and $\mathrm{Z}$ rotations and the detector ladders for $\mathrm{X}$ translations. The quality of the alignment has been validated with an independent data sample from the TED run by studying the mean (bias) and $\sigma$ (resolution) of the unbiased residual distributions per ladder. The $\sigma$ of the bias distribution $(15 \mu \mathrm{m})$ is an indication of the size of the misalignments that remain. The quality of the alignment can also be judged from the probability of the $\chi^{2}$ given by the track fit. This is shown in Figure 2 for the tracks passing through the IT boxes.

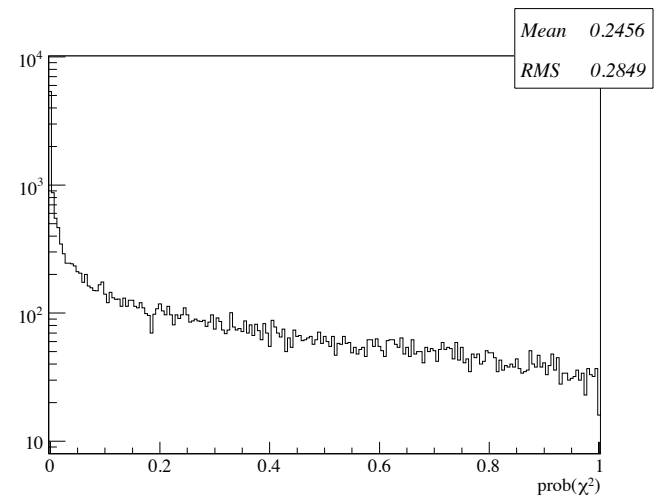

Figure 2: Probability of $\chi^{2}$ for tracks reconstructed in the IT after alignment.

Using clusters on the reconstructed tracks the $\mathrm{S} / \mathrm{N}$ has been studied. For each ladder a fit of a Landau convolved with a Gaussian is made and the Most Probable Value of the Landau extracted. Figure 3 shows the S/N per ladder obtained. For the long (short) ladders a $\mathrm{S} / \mathrm{N}$ of 14.5 (15.5) is obtained. The long ladders are thicker $(410 \mu \mathrm{m})$ than the short ladders $(320 \mu \mathrm{m})$. These values are consistent with expectations from test-beam measurements. Finally, the ladder efficiency has been measured to be $98 \%$ using the tracks. Most of the ladders with low efficiency are located close 
to the edge of the detector. Therefore, this number will improve when the detector alignment and global positioning is better understood.

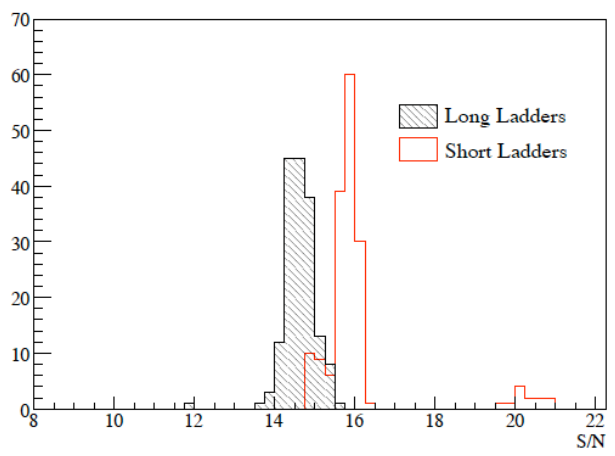

Figure 3: Signal to noise ratio of the Inner Tracker ladders.

In the case of the TT station standalone tracking is not possible since it has only four layers. Performance studies rely on track seeds either from the Vertex Locator (VELO) or the IT. Extrapolating tracks from the VELO and IT to the TT and calculating the residual to hits in TT clear correlations are seen. These distributions have allowed to verify the quality of the survey that was performed during the detector installation. As an example Figure 4 shows the residual between clusters in the TTb x-layer and tracks in the IT Bottom box. The width of the distribution $(1.1 \mathrm{~mm})$ is consistent with the tracks having a momentum of $10 \mathrm{GeV}$.

\section{Summary}

The data collected during the LHC synchronization tests has been used to study the performance of the Silicon Tracker. An initial alignment of the detector with straight tracks has been performed. The efficiency of the detector has been shown to be good. The knowledge acquired during this tests will definitely help understanding the detector with colliding beams at the end of 2009. 


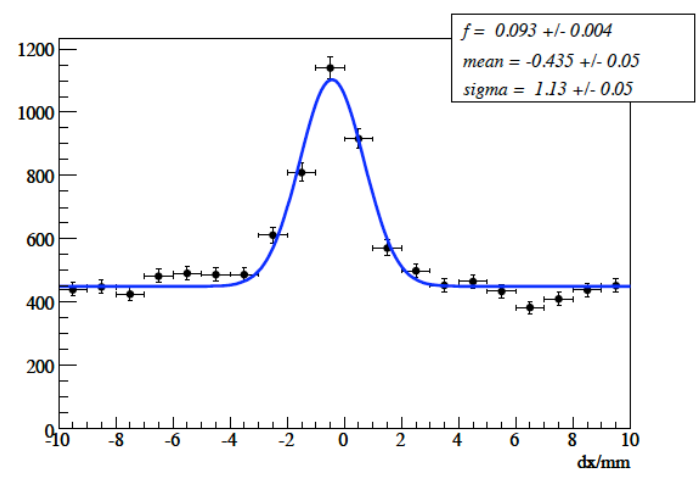

Figure 4: Residual of clusters in the TTb X to IT Bottom tracks. The data was fitted to Gaussian combined with a flat background.

\section{References}

[1] The LHCb Detector at the LHC. The LHCb collaboration. Journal of Instrumentation, 2008 JINST 3 S08005.

[2] H. Voss, The LHCb Silicon Tracker, Nucl. Instrum. Meth., vol. A549, pp. 44-48, 2005.

LHCb Reoptimized Detector Design and Performance Technical Design Report. CERN/LHCC-2003-030. ISBN 92-9083-209-6.

[3] N. Bakel et al., The Beetle Reference Manual, LHCb note 2001-046.

[4] A. Vollhardt et al., Production of the LHCb Silicon Tracker Readout Electronics, Proc. LECC 2005, CERN/LHCC-2005-038 187-191.

[5] Alignment of the Inner Tracker Stations Using First Data. L. Nicolas and M. Needham LHCb-PUB-009-012 